\title{
P I 7-04. Targeting HIV peptides to human dendritic cells via CD40 elicits expansion of multi-epitope polyfunctional CD4+ and CD8+ T cells in HIV patients
}

\author{
A Flamar*1, S Zurawski ${ }^{1}$, A Cobb ${ }^{1}$, B King ${ }^{2}$, L Sloan ${ }^{2}$, Y Levy ${ }^{3}$, J Banchereau ${ }^{1}$ \\ and G Zurawski ${ }^{1}$
}

\author{
Address: ${ }^{1}$ Baylor Institute for Immunology Research INSERM U899, Dallas, TX, USA, ${ }^{2}$ North Texas Infectious Disease, Dallas, TX, USA and \\ ${ }^{3}$ INSERM U481, ANRS Vaccine Programme, Faculte de Médecine de Créteil, Créteil, France \\ * Corresponding author
}

from AIDS Vaccine 2009

Paris, France. 19-22 October 2009

Published: 22 October 2009

Retrovirology 2009, 6(Suppl 3):P286 doi:10.1 186/1742-4690-6-S3-P286

This abstract is available from: http://www.retrovirology.com/content/6/S3/P286

(C) 2009 Flamar et al; licensee BioMed Central Ltd.

\section{Background}

Targeting Dendritic Cells (DCs) with anti-DC receptor antibody-antigen fusion proteins represents a novel approach to vaccine development. In mouse models, these innovative vaccines induce enhanced cellular, humoral, or mixed immune responses. Targeting antigens to particular DC subsets can lead to distinct immune outcomes but the consequence of targeting antigen via different receptors on the same DC is not well-studied.

\section{Methods}

We developed recombinant mAbs (rAbs) for multiple internalizing human DC receptors and engineered them fused via the heavy chain C-terminus to a string of five 19to 32-amino-acid long sequences from HIV-1 Gag, Nef and Pol proteins (rAb.HIV5pep). These peptides bear multiple highly conserved $\mathrm{CD} 4+$ and $\mathrm{CD} 8+\mathrm{T}$ cell epitopes. Anti-DCIR, anti-LOX-1, anti-CD40, and a control IgG4.HIV5pep fusion proteins were incubated with HIV patient PBMCs or DC-T cell co-cultures and after 10 days the total $\mathrm{T}$ cells were stimulated with each individual HIV peptide, and then antigen-specific cytokine production was detected using intracellular staining and multiplex bead assay from culture supernatants.

\section{Results}

At low doses $(10-0.01 \mathrm{nM})$ all three DC-targeting HIV5pep vaccines, but not the control IgG4.HIV5pep, elicited expansion of antigen-specific T cells. The range of antigen-specific $\mathrm{T}$ cells recall responses over the $5 \mathrm{HIV}$ peptide regions varied for each patient, but in sum epitopes from all 5 regions could be effectively processed and presented. All three DC-targeting vaccines expanded antigen-specific T cells, but anti-CD40.HIV5pep was strikingly more effective for eliciting multi-epitope CD4+ and CD8+ $\mathrm{T}$ cell responses. The expanded antigen-specific $\mathrm{T}$ cells were polyfunctional since the CD4+ T cells produced multiple cytokines, while the CD8+ T cells produced cytokines and had CTL characteristics (perforin, granzyme $\mathrm{B}$, surface CD107a, proliferation capacity).

\section{Conclusion}

Our in vitro data justify preclinical studies with antiCD40.HIV5pep in non-human primates for priming naive $\mathrm{T}$ cells with a broad range of epitopes and potent effector functions. 